\title{
Ein großes Dankeschön und ein herzliches Willkommen
}

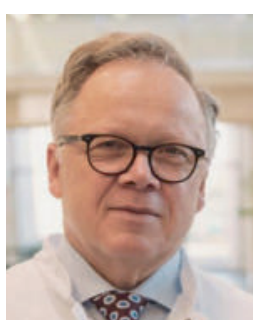

Prof. Dr.

Heinz-Jürgen Lakomek

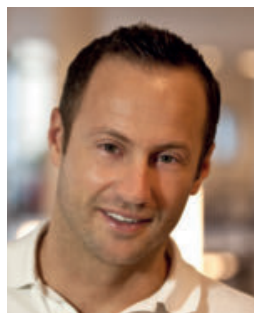

Priv.-Doz. Dr. Xenofon Baraliakos

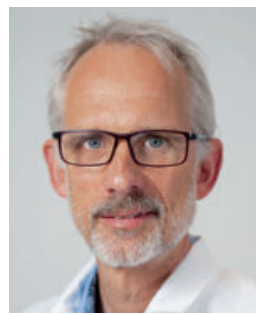

Prof. Dr. Christoph Fiehn

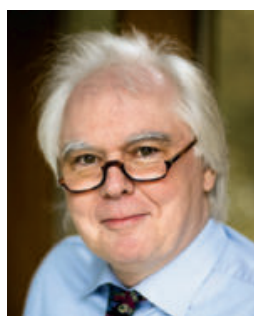

Prof. Dr.

Wolfgang Rüther

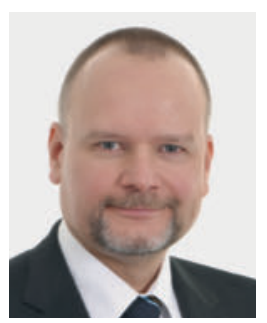

Prof. Dr. Ralph Gaulke
Unsere Zeitschrift wird sich abermals erneuern und verjüngen. Prof. Dr. med. Heinz-Jürgen Lakomek gibt nach 12 Jahren als Herausgeber der arthritis + rheuma den Staffelstab weiter an Priv.-Doz. Dr. med. Xenofon Baraliakos.

H.-J. Lakomek hat seit 2008 die Geschicke der Zeitschrift entscheidend gestaltet. Alle, die diese Zeit in der Rheumatologie erlebt haben, wissen, dass sich seit 2008 wesentliche Dinge verändert haben. Dies meint nicht nur die neuen medikamentösen Therapieoptionen und -strategien, die, mit der rheumatoiden Arthritis beginnend, inzwischen die allermeisten rheumatologischen Krankheitsbilder betreffen. Es sind auch die Rahmenbedingungen der Versorgung der Menschen mit rheumatischen Erkrankungen, die seitdem ständig im Fluss waren und weiterhin sind. Stellvertretend sei die Einführung und Etablierung der ambulanten spezialärztlichen Versorgung (ASV) zu nennen. H.- J. Lakomek hat bei diesen Veränderungen wesentlich mitgewirkt.

Insgesamt 13 Ausgaben der arthritis + rheuma hat er in dieser Zeit persönlich als Herausgeber gestaltet und viele mehr thematisch angestoßen. Das Spektrum der Themen spiegelt die jeweils aktuellen Herausforderungen der Rheumatologie wider. Sein Anspruch - und der unserer Zeitschrift - war immer, den aktuellen Stand der Medizin möglichst praktisch relevant und mit Fortbildungsfunktion, zu beschreiben. Seit 2009 ist die arthritis + rheuma das offizielle Mitteilungsorgan des Verbandes der rheumatologischen Akutkliniken (VRA), dessen Vorstandsvorsitzender H.-J. Lakomek über viele Jahre war und dessen jetziger Geschäftsführer er seit Ende 2019 ist.

Priv.-Doz. Dr. med. Xenofon Baraliakos (44 J.) aus Herne ist leitender Oberarzt am Rheumazentrum Ruhrgebiet und Facharzt für Innere Medizin/Rheumatologie sowie für Orthopädie. Er ist an der Ruhr-Universität Bochum habilitiert und als international bekannter Experte auf dem Gebiet der Spondyloarthritiden auch Autor einer Vielzahl hochrangiger Publikationen auf dem Gebiet. X. Baraliakos, der für seine Wissenschaftsleistung diverse Preise unter anderem der EULAR erhalten hat, ist seit Januar 2020 auch Präsident der internationalen Gesellschaft für die Erforschung der Spondyloarthritiden (Assessment of SpondyloArthritis international Society, ASAS). Wir freuen uns ganz besonders, dass wir ihn trotz dieser vielen Aufgaben dafür gewinnen konnten, die Herausgeberschaft der arthritis + rheuma mit zu übernehmen.

Wir und das Redaktionsteam der arthritis + rheuma danken H.-J. Lakomek ganz herzlich für seine unglaublich stetige und fruchtbare Tätigkeit als Herausgeber in den vergangenen Jahren und wünschen ihm für die Zukunft alles Gute. Darüber hinaus freuen wir uns sehr auf die zukünftigen Projekte mit X. Baraliakos als seinem Nachfolger.

Ihre

Prof. Dr. med. Christoph Fiehn

Prof. Dr. med. W. Rüther

Prof. Dr. med. R. Gaulke 